LBNL - 63420

\title{
Demand Controlled Filtration in an Industrial Cleanroom
}

\author{
David Faulkner, Dennis DiBartolomeo, Duo Wang \\ Environmental Energy Technologies Division \\ Indoor Environment Department \\ Lawrence Berkeley National Laboratories \\ Berkeley, CA 94720
}

September 2007

This work is governed under U.S. Department of Energy Contract No. DE-AC0376SF00098 with the Regents of the University of California and funded by the California Energy Commission's Public Interest Energy Research (PIER) Program through agreement with the Regents of the University of California administered by the California Institute for Energy and Environment (CIEE) (under Memorandum Agreement NO. MR0312). 


\title{
Demand Controlled Filtration in an Industrial Cleanroom
}

David Faulkner, Dennis DiBartolomeo, Duo Wang

\begin{abstract}
In an industrial cleanroom, significant energy savings were realized by implementing two types of demand controlled filtration (DCF) strategies, one based on particle counts and one on occupancy. With each strategy the speed of the recirculation fan filter units was reduced to save energy. When the control was based on particle counts, the energy use was $60 \%$ of the baseline configuration of continuous fan operation. With simple occupancy sensors, the energy usage was $63 \%$ of the baseline configuration. During the testing of DCF, no complaints were registered by the operator of the cleanroom concerning processes and products being affected by the DCF implementation.
\end{abstract}

\section{Introduction}

Demand controlled filtration (DCF) is a method of saving energy in cleanrooms by decreasing the recirculation fan speed when demand for filtration is low. A particle counter or an occupancy sensor can measure the demand for filtration. Another method of saving energy in cleanrooms is the use of programmable timers that decrease recirculation fan speeds when occupants are not expected, such as nights and weekends.

DCF should not be confused with demand controlled ventilation, which is the control of outside air entering a building based on the demand due to occupancy and/or processes occurring in the building.

In a past study, we found that industrial cleanroom users were hesitant to implement DCF, often because of concern that large changes in the recirculation fan speeds would produce a burst of particles discharged into the cleanroom. We have not seen this phenomenon in our previous or current study. Another concern is that for large manufacturing facilities, the potential loss of product outweighs any potential energy savings. There are many small research or industrial facilities that are not as productoriented that can benefit from the potential energy savings from implementing DCF.

In the past few years there has been gathering interest in saving energy in cleanrooms and some businesses are experimenting with DCF. There is a large Southern California manufacturer with Class 100 (ISO Class 5) and 10,000 (ISO Class 7) cleanrooms covering about 15,000 $\mathrm{ft}^{2}$. During non-working hours, the make-up and recirculation air handler fans at this facility are turned down to recirculate less air. A reduction in fan speed of about $30 \%$ at night, when the space is unoccupied, reduces the measured recirculation air handler power consumption from $32 \mathrm{~kW}$ to less than $9 \mathrm{~kW}$ - a reduction of $75 \%$.

Another large manufacturing facility in Southern California is in the process of implementing active, real-time DCF using particle counters. The facility manager has received rebates from the local utility for implementing DCF. The particle counters have been purchased and preliminary testing has been done. The next steps are installing the particle counters and implementing the control routines. 
Finally, a large East Coast university recently modernized a building with cleanrooms. In the process they installed particle counters in every cleanroom area and plan to implement DCF.

\section{Energy savings potential}

The potential for energy savings is tremendous as fan energy has an approximately cubic relationship with fan speed. Thus, even a small reduction in fan speed can translate into large energy savings. Cleanrooms employing DCF can achieve lasting savings by implementing active control of the recirculation fans based on inputs from either a particle counter or an occupancy sensor.

Also, cleanroom operators may be able to participate in demand response programs offered by utilities. During high electricity demand days, such as hot summer days, many utilities offer incentives to their customers, such as rebates or price reductions, to voluntarily reduce electricity demand at their facilities. Typically these programs ask customers to reduce electricity consumption for periods of 1-6 hours. As long as the cleanroom fans are equipped with variable frequency drives (VFD), then the recirculation fan speeds can be adjusted lower during critical demand days. A 10-20\% reduction in recirculation fan speed can translate to a reduction in energy use of over $40 \%$.

\section{Methods}

Facility

Experiments were conducted to evaluate air quality in an industrial cleanroom in the San Francisco Bay Area while DCF was implemented. The cleanroom that was studied was one of a suite of four adjacent cleanrooms, each with different classifications from Class 10 (ISO Class 4) to Class 10,000 (ISO Class 7). The suite of cleanrooms was designed so that the inner Class 10 (ISO Class 4) cleanroom (Group-5) is at a higher pressure than the adjacent Class 100 (ISO Class 5) cleanroom (Group-6), which in turn is at higher pressure than the adjacent Class 1,000 (ISO Class 6) cleanroom (Group-3), etc., see Figure 1. There are open grilles for air to flow from the Group-5 cleanroom to the Group-6 cleanroom to the Group-3 cleanroom and finally to the Group-2 cleanroom. The cleanroom in which the experiments were conducted was the Group-2 area and was a Class 10,000 (ISO Class 7).

The floor area of the entire suite is about $1600 \mathrm{ft}^{2}$ and the floor area of the Group-2, the experimental cleanroom, was $600 \mathrm{ft}^{2}$. The layout of Group-2 is shown in Figure 2. Each cleanroom had air recirculated by ceiling-mounted $2 \mathrm{ft} \times 4 \mathrm{ft}$ fan filter units with HEPA filters. The Group-2 cleanroom had 16 fan filter units covering about $20 \%$ of the ceiling area. There were 4 hoods in the middle of the cleanroom as well as 2-3 flow hoods at each end of the cleanroom. Airflow through these hoods were not controlled as a part of our study.

Outside make-up air was controlled by a separate ventilation system and was not controlled in our study. Environmental conditions, temperature and humidity were controlled by a separate control and also were not part of our study. 
Before our study, a laptop computer using a proprietary software program controlled the cleanroom fan filter units. The software allowed control of the fan filter units individually or in groups. This software was used to lower the fan speeds to $50 \%$ at night (22:00 to 6:00) and on weekends. This software and corresponding laptop would occasionally fail and the fan filter units would run at the speed of the last valid command; the fan filter units are designed to always run at the last valid command. Thus, occasionally the fan filter units for all of the cleanrooms would run for several days at $50 \%$ speed before the problem was discovered.

After implementing the night and weekend energy reduction, particle concentrations at 4 locations in the cleanroom suite were recorded continuously for background data. The particles concentrations were measured with MetOne 237B particle counters (Hach Ultra, Grants Pass, OR) capable of counting particles in 6 size bins from 0.3 to 5 microns at a sample flow rate of $0.1 \mathrm{cfm}$. Some particle counters were moved to different locations within the cleanroom suite during the background measurements. Based on this data and the desires of the facility manager, the experiments were performed in the Group- 2 cleanroom which was the Class 10,000 (ISO Class 7) cleanroom.

After background particle measurements were recorded, a particle counter was placed in the study cleanroom (Group-2) near the center of the room on a countertop about $3 \mathrm{ft}$ above the floor. To implement DCF, the particle counter was connected to a laptop computer running an off-the-shelf software program, LabVIEW Version 5.1 (National Instruments). Control routines in LabVIEW had been developed to record particle counts, and use the particle count information to decide whether to increase or decrease fan speeds. The particle counter sent counts to the laptop every 30 seconds and the particle counts in the 0.3 and 0.5 micron size bins were compared to established upper limits. The fan speed was either increased or decreased until either the maximum or minimum fan speed was reached. A record of the fan speed, particle counts and other parameters were recorded every 60 seconds in LabVIEW.

After implementing DCF with the particle counter, we implemented DCF with control based on occupancy. The strategy was simple; if there were occupants detected, then the fan filter units were set to the programmed maximum speed, and after 30 minutes of no occupancy detected, the fans were set to $50 \%$ of programmed maximum speed.

There were 6 wireless infrared battery-operated occupancy sensors placed in the cleanroom. The 6 sensors were mounted on the walls so that each work area and traffic lane was covered by at least one occupancy sensor. A wireless signal was sent from the occupancy sensors to the laptop and the above strategy was implemented.

The data from the particle counter was converted to an equivalent ISO Class using the following equation (ISO 14644-1: 1999(E)):

$$
C_{n}=10^{N}\left(\frac{0.1}{D}\right)^{2.08}
$$


where,

$C_{n}$ is the maximum permitted concentration (in particles per cubic meter of air) of airborne particles that are equal to or larger than the considered particle size. Value is rounded to the nearest whole number, using no more than three significant figures.

$N$ is the ISO classification number, which shall not exceed a value of 9 .

Intermediate ISO classification numbers may be specified; with 0.1 the smallest permitted increment of $N$.

$\mathrm{D}$ is the considered particle size, in micrometers, which was $0.3 \mu \mathrm{m}$ in this study.

0.1 is a constant, with a dimension of micrometers.

With algebraic manipulation equation (1) can be rearranged to solve for $\mathrm{N}$, the ISO classification number:

$$
N=\log _{10} C_{n}+2.08\left(1+\log _{10} D\right)
$$

\section{Results}

The data presented in Figures 3-6, show the ISO Class for the particle size of $D=0.3 \mu \mathrm{m}$ when the Group-2 cleanroom was using DCF controlled by particle counts and by occupancy sensors. The concentration, $C_{n}$, is calculated from the counts measured by the particle counter.

During these experiments, the fan filter units were running at about $77 \%$ of maximum speed. Thus, in the following discussion, full speed means $77 \%$ of maximum speed and half speed means about 38\% of maximum speed. Also, in all plots, the greatest fan speed will be $77 \%$.

Figure 3 shows fan speed and particle counts in the Group-2 cleanroom (converted to ISO Class) versus time for a weekday while the fan speed was controlled by particle counts. From the graph, it is clear that the fans are actively being controlled by the particle counts most of the work day from about 6:00 to 17:00. The ISO Class stays around 3.5 to 4 during the working hours. At night the particle counts increase, despite there being no occupants in the cleanroom. The reason for this increase in particle counts while there were no occupants has not been explained by the building manager/cleanroom operator. Pressure measurements made between cleanrooms showed that at night, some of the pressure differences between cleanrooms reduced in magnitude and some actually reversed direction. It was thought that this was possibly due to changes in the whole building HVAC system. The building manager did not have details concerning nighttime setback conditions in the whole building HVAC system. Each morning, as the whole building HVAC system reset and the cleanroom fan filter units increased, the cleanroom, returned to "clean” conditions very quickly.

Figure 4 shows the fan speed and particle count data for a weekday while the cleanroom had DCF control based on occupancy sensors. The fan filter units were either at full speed (77\% of maximum) while occupants were detected or at "half" speed ( 38\% of 
maximum) with no occupants detected in the last 30 minutes. After noon, it can be seen that no occupants were detected on 2 or 3 occasions. In response, the fan filter units reduced to half speed and the ISO Class (based on particle counts) increased rapidly from about 3 to 5 . But each time occupants were detected, the particle counts decreased quickly.

The rapid response in particle counts to the change in fan filter speed is seen in Figure 5, which shows similar data as in Figure 4, but over a shorter time span. The ISO Class changes from about 5 to about 3 in approximately 3-4 minutes as the fan speed increased from half speed to full speed.

Finally, Figure 6 shows the fan speed and particle count for a weekend day with DCF control based on occupancy sensors. As soon as an occupant was detected around 9:00, the fan speed increased to full speed and the particle counts decreased by about a factor of 300 (ISO Class 6 to Class 3.5). Around 10:10, an absence of occupants was detected and fan speeds were set to "half" speed and the particle counts increased. About 10 minutes later, an occupant was detected and the fan speeds increased to "full" speed and particle counts again decrease, as indicated by the ISO Class.

Energy values are shown in Table 1, for a typical week. There are 5 different configurations that are compared. Two of the configurations are used as baselines for comparison with various energy saving configurations. The baselines are $24 / 7$, which means the fans are running all the time, and the other baseline is the night and weekend setback, in which the fans were reduced at night and on the weekends. Comparing the two baselines, the night and weekend setback saves about $28 \%$ in energy as compared to the fans running constantly.

The use of the particle counter to implement DCF saved more than the occupancy sensors, but not by much, $40 \%$ vs $36 \%$ as compared to $24 / 7$ as a baseline and $17 \%$ vs. $12 \%$ as compared to the night and weekend setback as a baseline. Thus, using a few occupancy sensors at $\$ 30$ each is less expensive than a particle counter that may cost $\$ 3,000$ or more.

Table 1. Energy use (kW-h per week) and percent savings due to demand controlled filtration.

\begin{tabular}{|l|c|c|c|}
\hline \multicolumn{1}{|c|}{ Configuration } & $\mathbf{k W}$-h per week & $\begin{array}{c}\text { Compared to } \\
\mathbf{2 4 / 7}\end{array}$ & $\begin{array}{c}\text { Compared to Night } \\
\text { \& Weekend Setback }\end{array}$ \\
\hline $24 / 7$ & 151 & & \\
\hline Night \& Weekend Setback & 109 & $28 \%$ & $12 \%$ \\
\hline Occupancy Sensor & 96 & $36 \%$ & $17 \%$ \\
\hline Particle Counter & 91 & $40 \%$ & $36 \%$ \\
\hline "Half" Speed & 70 & $54 \%$ & \\
\hline
\end{tabular}




\section{Conclusions}

The data collected indicate two major conclusions. First, DCF was implemented without disruption to the processes in the cleanroom. This implementation was relatively easy and inexpensive, less than $\$ 10,000$ in equipment. Second, recovery of particle concentration from a "dirty" condition to a "clean" condition occurred in a matter of minutes. Thus, those cleanroom operators in industry need not be concerned about the removal of particles taking days or hours after a reduction in the fan speeds.

The energy savings realized were about $40 \%$ with either method as compared to running the cleanroom fans constantly, 24/7. Savings were about $15 \%$ with either method as compared to night-time and weekend setback.

\section{Acknowledgements}

Bill Tschudi and Mark Sippola of Lawrence Berkeley National Lab, Larry Chu and Peter Rumsey of Rumsey Engineers, and Reza Kazemi of Environmental Filtration, Inc.

\section{References}

Faulkner, D., Fisk, W.J., and Walton, J.T. (1996) Energy Savings in Cleanrooms from Demand-Controlled Filtration”, Journal of the Institute of Environmental Sciences 39(2): 21-27. LBNL-38869

International Standard, Cleanrooms and associated controlled environments - Part 1: Classification of air cleanliness, ISO 14644-1: 1999(E) 


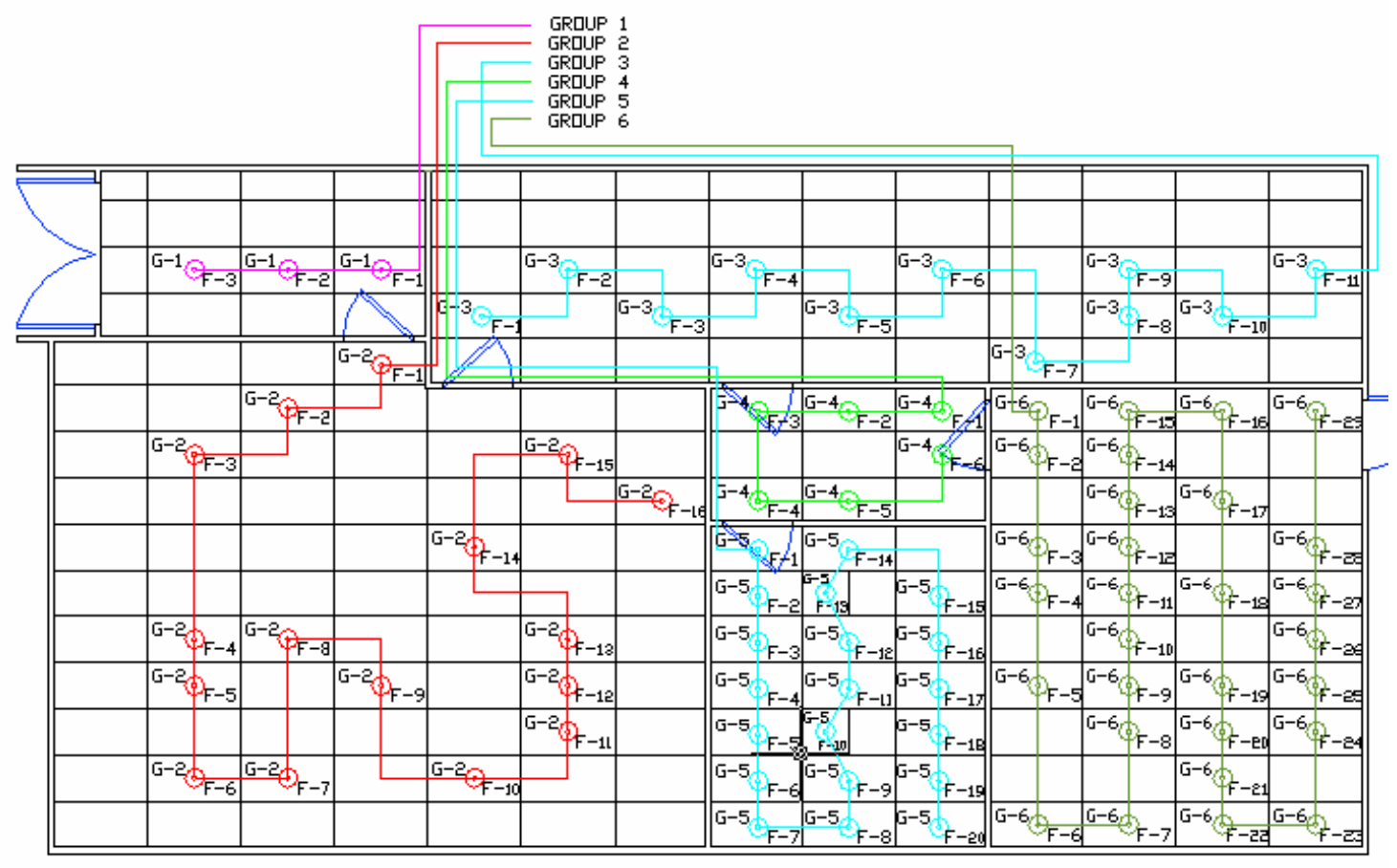

Figure 1. Layout of the suite of cleanrooms in the studied industrial facility. G-5 (Group-5) is an ISO Class 4 cleanroom. G-2 (Group-2) is an ISO Class 7 cleanroom. G1 (Group-1) and G-4 (Group-4) are gowning rooms. G-3 (Group-3) is an ISO Class 6 cleanroom and G-6 (Group-6) is an ISO Class 5 cleanroom. Fan filter units are labeled as $\mathrm{F}-1, \mathrm{~F}-2$, etc. Each rectangle is $2 \mathrm{x} 4 \mathrm{ft}$. 


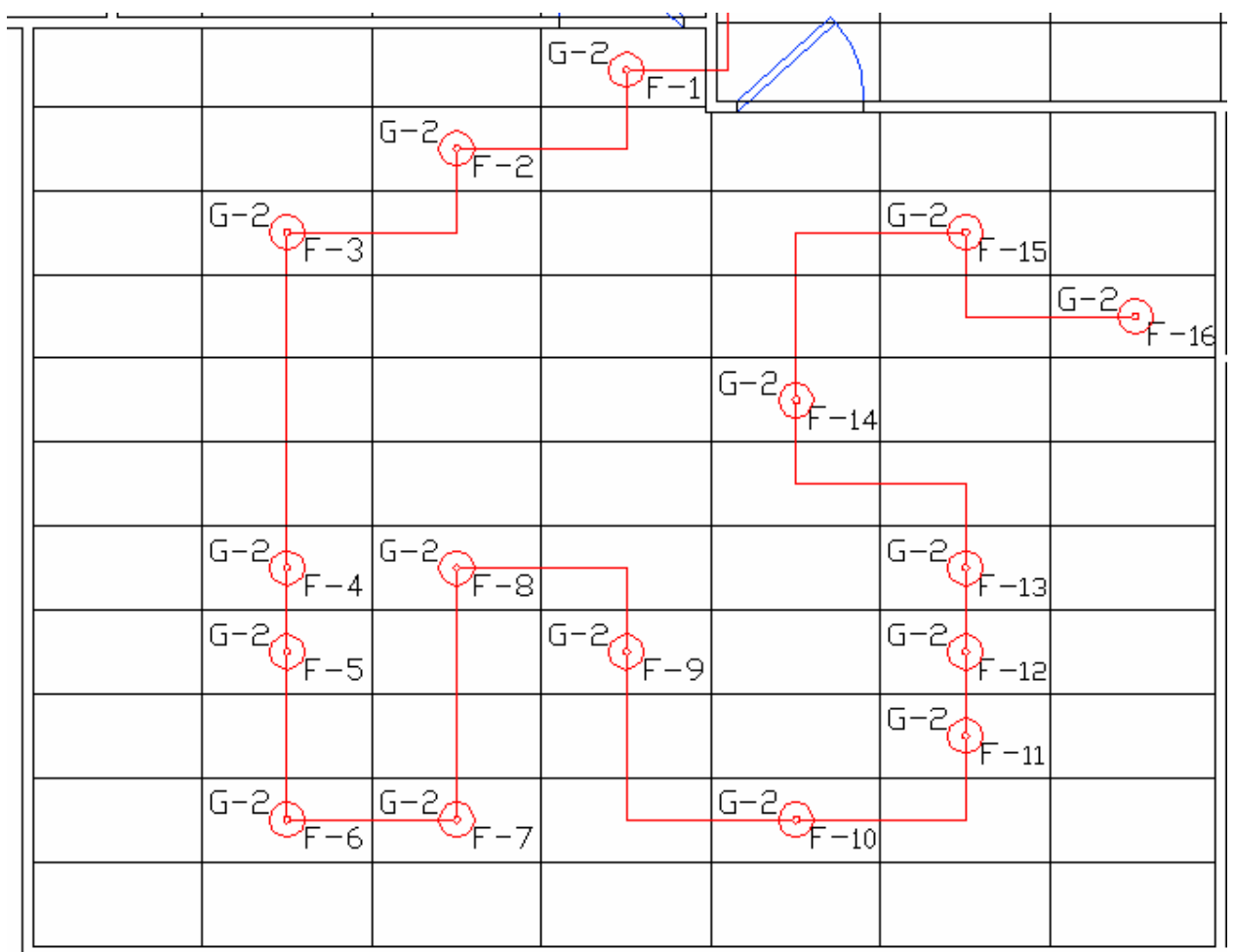

Figure 2. Layout of fan filter units in study cleanroom, Group-2. There are 16 fan filter units, shown as F-1 to F-16. Each rectangle is 2 x $4 \mathrm{ft}$.

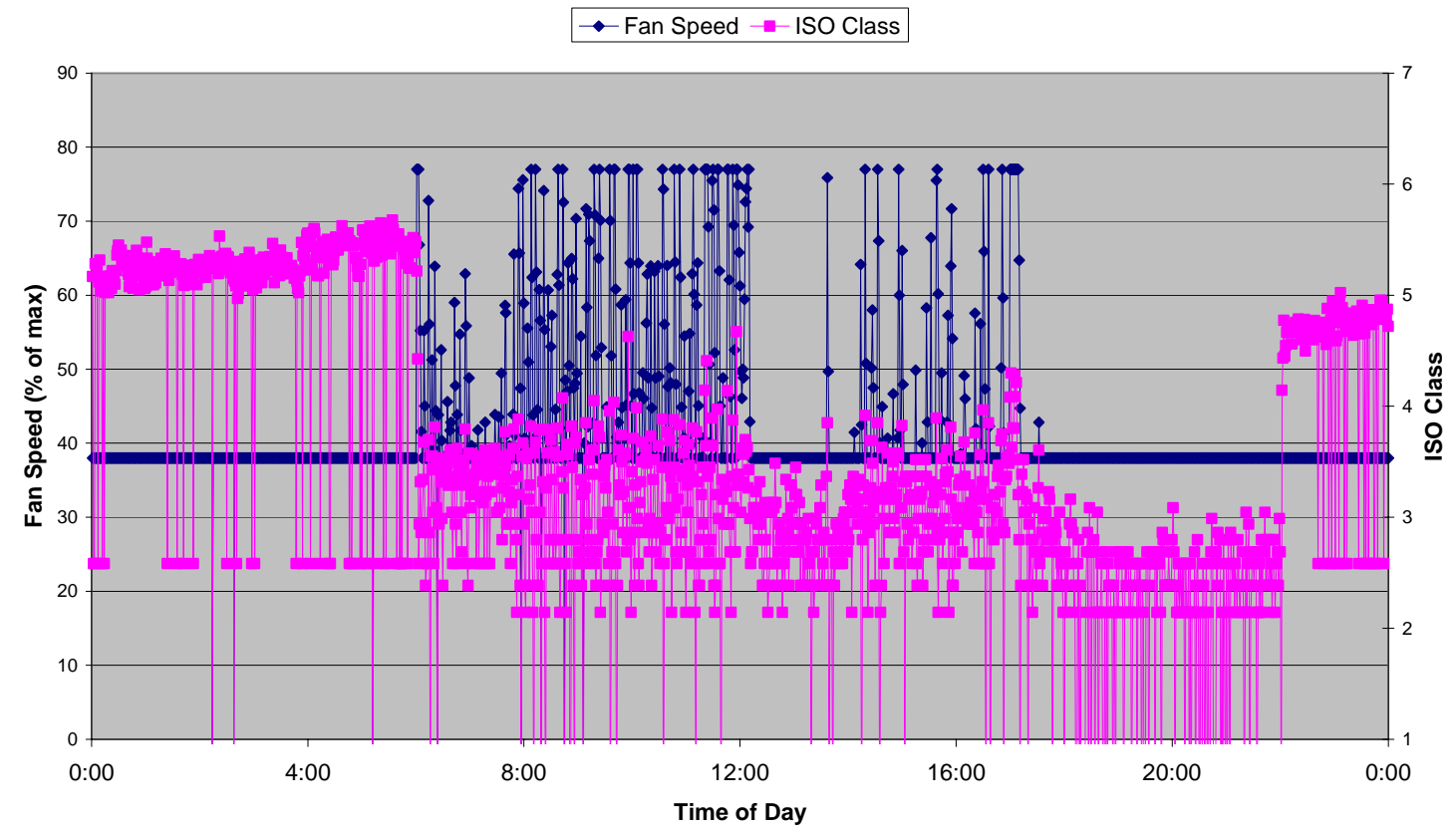

Figure 3. Fan speed and particle counts (converted to ISO Class) versus time for a weekday with DCF control based on particle counts. The cleanroom classification is based on the particle concentration at 0.3 micrometers. 


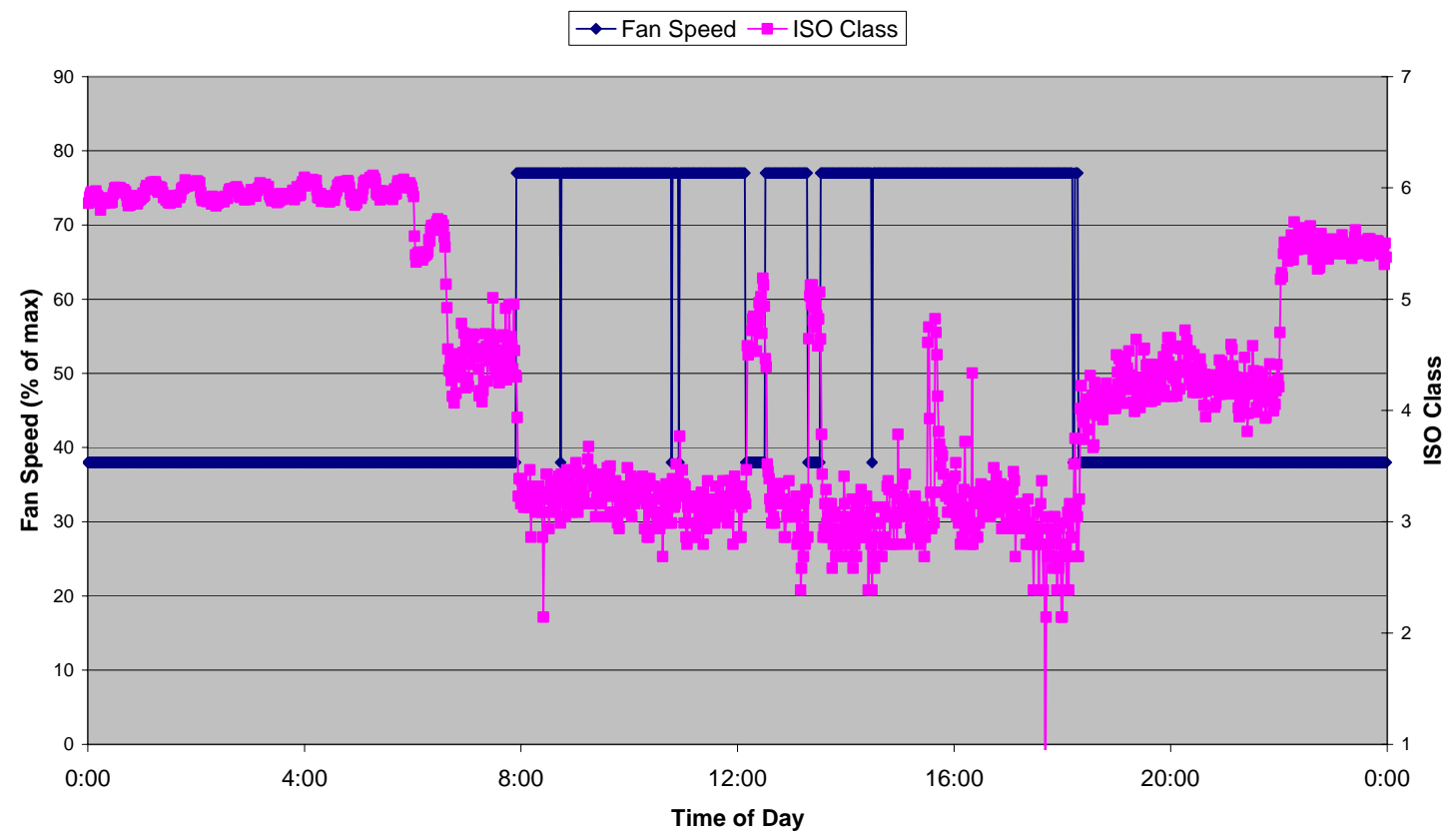

Figure 4. Fan speed and particle counts (converted to ISO Class) versus time for a weekday with DCF control based on occupancy sensors. The cleanroom classification is based on the particle concentration at 0.3 micrometers.

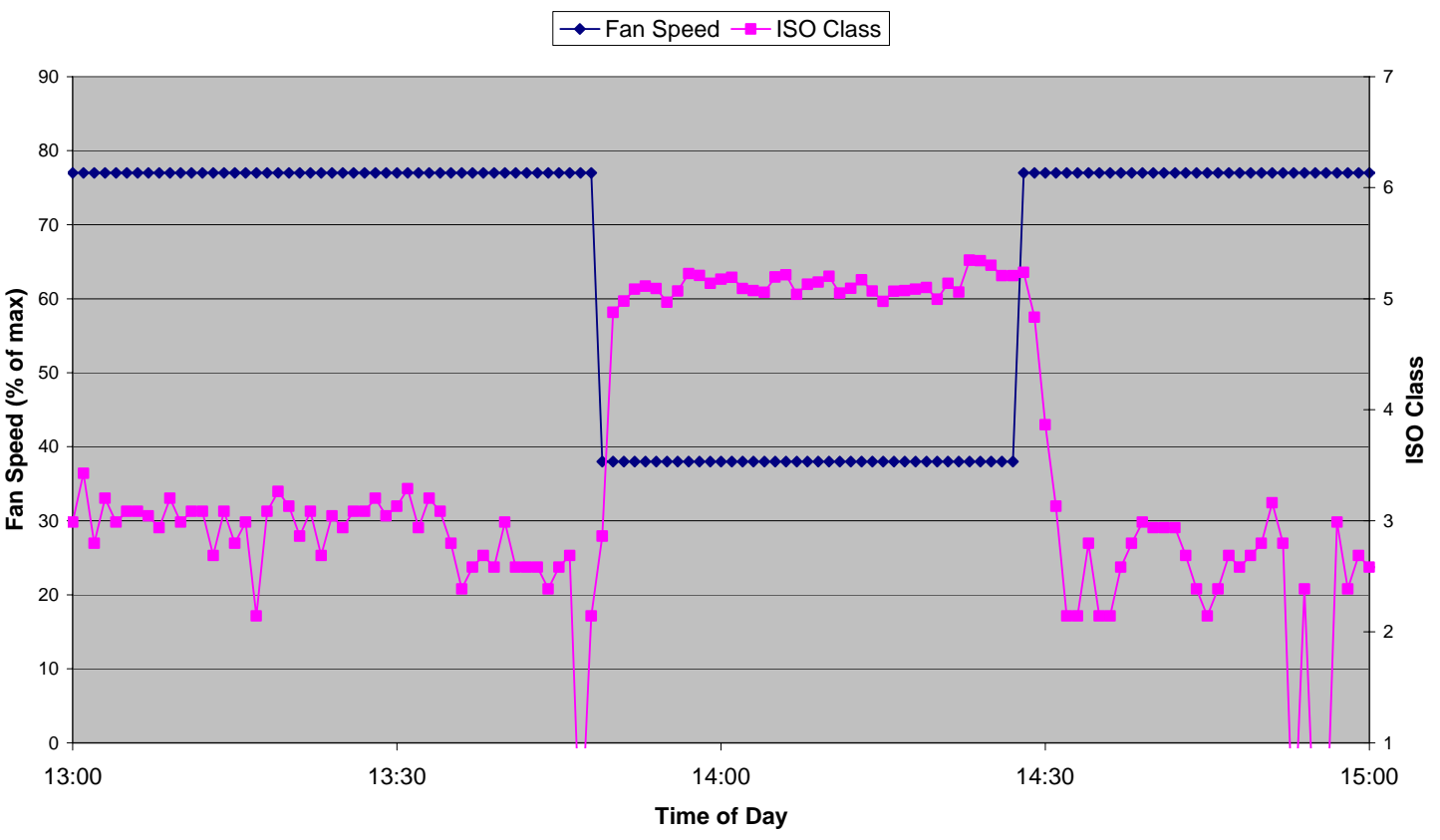

Figure 5. Fan speed and particle counts (converted to ISO Class) versus time for a weekday with DCF control. There is a rapid return to "clean" conditions within 3-4 minutes (data recorded every 1 minute) after the fans increase from a low speed to a high speed. The cleanroom classification is based on the particle concentration at 0.3 micrometers. 


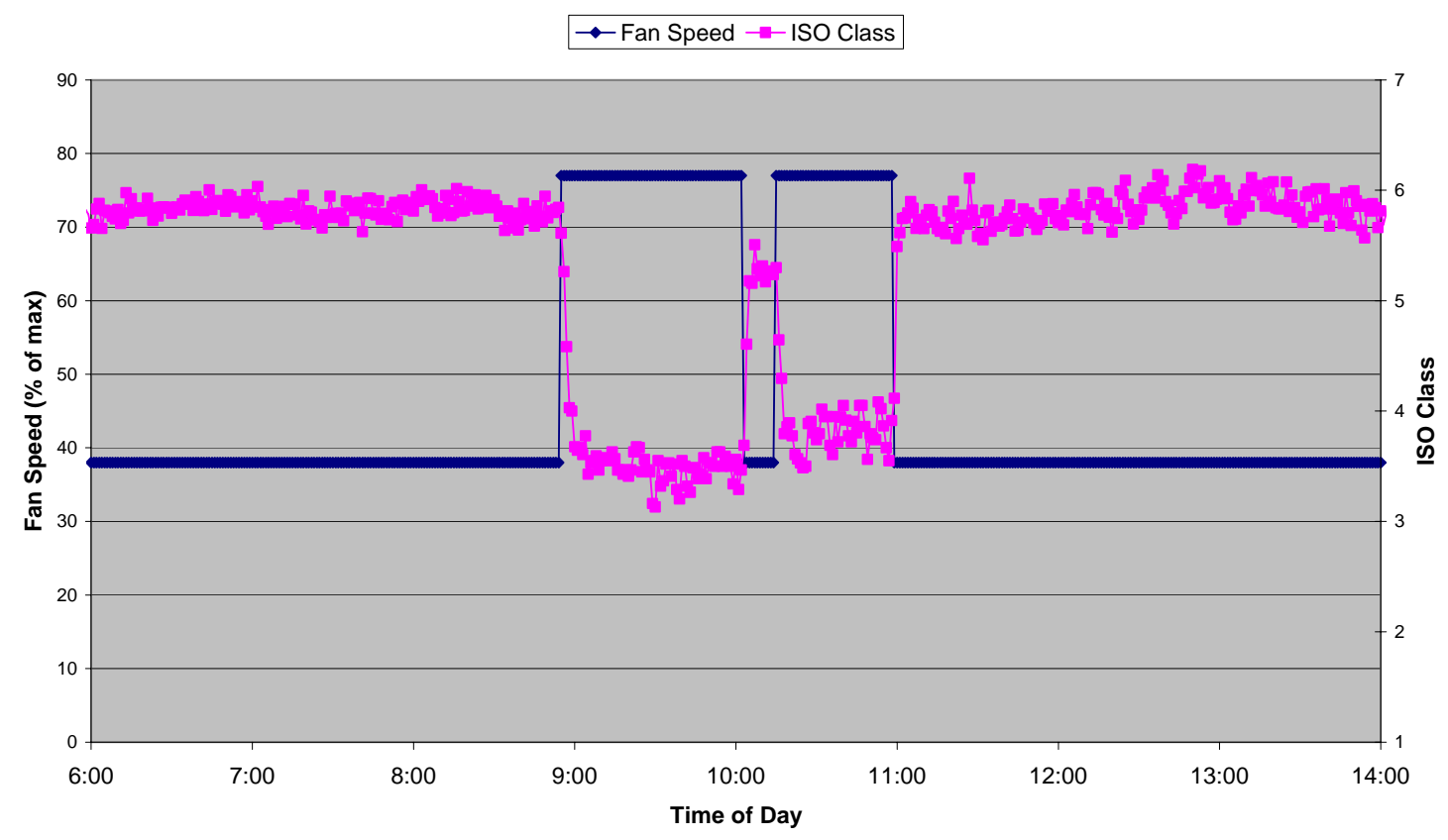

Figure 6. Fan speed and particle counts (converted to ISO Class) for a weekend day with DCF control based on occupancy sensors. Changes in particle concentrations, as expressed by the ISO Class, are very quick. The cleanroom classification is based on the particle concentration at 0.3 micrometers. 